Artículo

\title{
Vegetación y estructura del hábitat que determina la dieta de aves insectívoras en sistemas agroforestales
}

\author{
Rosa María García-Núñez ${ }^{1}$ \\ Claudio Romero-Díaz ${ }^{1}$ \\ Saúl Ugalde-Lezama ${ }^{2 \S}$ \\ Juan Ángel Tinoco-Rueda ${ }^{3}$ \\ ${ }^{1}$ Maestría en Ciencias en Agroforestería para el Desarrollo Sostenible-Universidad Autónoma Chapingo. \\ Carretera Federal México-Texcoco km 38.5. Texcoco, México. CP. 56230. (blondynunez@gmail.com; \\ favia_rd@hotmail.com). ${ }^{2}$ Departamento de Suelos-Área de Recursos Naturales Renovables-Universidad \\ Autónoma Chapingo. Carretera Federal México-Texcoco km 38.5, CP. 56230. Texcoco, México. ${ }^{3}$ Centro \\ Regional Universitario Oriente-Universidad Autónoma Chapingo. Carretera Huatusco-Jalapa km 6, \\ Huatusco, Veracruz. CP. 94100. (tinoco@correo.chapingo.mx).
}

Autor para correspondencia: biologo_ugalde@ hotmail.com.

\section{Resumen}

Para definir la vegetación y estructura del hábitat con relación a la presencia de aves y presas de agosto 2018 a enero 2019 se llevó acabo el seguimiento de aves, vegetación e insectos, empleando recuento en puntos con radio fijo; búsqueda intensiva; corte y sacudida de ramas, líneas de Canfield, cuadrantes con punto central y el vecino más cercano. Se determinó frecuencia de observación (FO) e índice de abundancia relativa (IAR). Para determinar diferencias y conocer si lo registrado es lo esperado se desarrollaron análisis de kruskal wallis y $\mathrm{X}^{2}$. Para evaluar asociación entre el hábitat y vegetación en relación con la presencia de aves e insectos se aplicó un análisis de regresión Poisson (ARP). Para conocer el grado de inercia entre hábitat y vegetación en respuesta a la presencia de aves e insectos se desarrollaron análisis de correspondencia canónica (ACC). La FO señala valores altos para: Aves Melanerpes aurifrons, Cyanocorax morio y Empidonax sp.; vegetación Poaceae, Selaginellaceae y Verbenaceae; insectos Plecia nearctica y Chauliognathus pensylvanicus. El IAR señala valores altos para: vegetación Poaceae, Selaginellaceae y Verbenaceae; insectos Plecia nearctica y Chauliognathus pensylvanicus. Kruskal wallis sin diferencias y $\mathrm{X}^{2}$ que lo registrado es lo esperado. El ARP sugiere un $\mathrm{AIC}=283.65 ; 219.4 ; 240.38$. El ACC evidencia una inercia de: $66.26 \%$; 78.54\%; $93.89 \%$ y $84.62 \%$. La vegetación y estructura del hábitat son factores determinantes en la abundancia de aves, disponen del stock alimenticio que garantiza la conservación y equilibrio ecológico de los sistemas agroforestales.

Palabras clave: alimentación, avifauna, cobertura-arbórea, conservación, muestreo-sistemático.

Recibido: marzo de 2020

Aceptado: mayo de 2020 


\section{Introducción}

A nivel mundial se registran un total de 10500 especies de aves (Navarro-Sigüenza et al., 2014). Sin embargo, debido a una serie de acciones antrópicas se presentan efectos negativos crecientes que colocan en riesgo la supervivencia de ecosistemas y el hábitat de varios organismos (RamírezAlbores, 2009; Vázquez-Perez et al., 2009). Tales acciones han ocasionado la extinción de varias especies de aves y muchas de ellas se encuentran amenazadas (Cruz y Lauro, 2006).

México registra un total de 1076 especies de aves, de las cuales 102 son endémicas (Valencia-Trejo et al., 2014). Asimismo, la causa de una serie de eventos antrópicos se ha deteriorado drásticamente su ambiente natural poniendo en punto crítico su supervivencia (Almazán-Núñez et al., 2009). La abundancia y distribución de aves es un reflejo de los componentes históricos y ecológicos. Dentro de los factores ecológicos se destaca la estructura del hábitat y recurso alimenticio (Cuento, 2006).

La vegetación que define la diversidad avifaunistica se ve transformada en base a una escala espacial integrada a nivel de hábitat y paisaje y una escala temporal que determina el acervo alimenticio (Buitrón-Jurado y Tobar, 2007). Sin embargo, la percepción de como dicho disturbio espacio temporal aqueja a diferentes organismos es aún incipiente (Naranjo y Ulloa, 1997).

A pesar de tener estudios sobre el movimiento horizontal de algunas aves en busca de mejores escenarios alimenticios y movimientos verticales de ciertos estratos, los estudios de estas fluctuaciones son muy limitados (Rangel-Salazar et al., 2009). El número de especies que habiten cierta región va a depender de tres factores importantes: disponibilidad de alimento, áreas de nidación y depredación a la que pudieran verse expuestas (Serial y Grigera, 2005).

Por su parte los sistemas agroforestales son una alternativa que favorece a la disponibilidad de hábitat para diferentes especies (Abouhamad et al., 2017). Teniendo un principio conservacionista, bajo un enfoque sostenible (Tzuc-Martínez et al., 2017; García, 2018). La estructura de estos sistemas está integrada por componentes (herbáceo, arbóreo y arbustivo) verticales y horizontales que simulan un ambiente natural favoreciente a la conservación de especies faunísticas.

En donde las aves pudieran encontrar un nicho trófico que le garantice su supervivencia (Castillo y Calderón, 2017; López-Ferrer et al., 2017). Un sistema agroforestal de café inmerso en bosque mesófilo de montaña (BMM), se ubica en el Municipio de Huatusco, Veracruz. Dicho sistema está conformado por diferentes especies arbóreas en el cual podrían habitar una gran diversidad de aves.

No obstante, a pesar de la importancia que representa este sistema en función de la conservación de especies, hasta el momento no se tienen estudios realizados en donde se aborde esta temática. El objetivo de este trabajo fue definir la relación en la presencia de aves e insectos presa con la vegetación y estructura del hábitat.

\section{Materiales y métodos}

Área de estudio. Se ubica entre las coordenadas geográficas $19^{\circ} 09^{\prime}$ de latitud norte y $96^{\circ} 57^{\prime}$ de longitud oeste a una altitud de 1933 msnm, perteneciente al municipio de Huatusco, Veracruz, México. Para dicho estudio se consideraron tres condiciones a evaluar: café tradicional (CT); potrero (PT) y bosque mesófilo de montaña (BMM) en una superficie total de 32.42 ha. 
Muestreo y toma de datos. En cada condición evaluada se aplicó un muestreo sistemático a conveniencia con distancias lineales de $150 \mathrm{~m}$ entre cada punto. El seguimiento de aves se realizó mensualmente de agosto 2018 a enero 2019 empleando recuento en puntos con radio fijo y búsqueda intensiva (Ponce et al., 2012; Alonso et al., 2017).

Paralelamente se aplicaron líneas de Canfield para determinar las variables del hábitat (Bueno et al., 2015) cuadrantes con punto central para vegetación herbácea y el vecino más cercano para vegetación arbórea (Mora et al., 2019). Aunado al recuento en puntos con radio fijo se empleó corte y sacudida de ramas, esta metodología consistió en que el investigador al ubicar a un ave alimentándose coloco una bolsa de plástico sobre la rama en la cual se encontraba el ave y sacudió para colectar a los insectos de los que potencialmente se pudiera estar alimentando esta ave.

Análisis de datos. Se determinó frecuencia de observación (FO) para aves, vegetación e insectos. Índice de abundancia relativa para vegetación e insectos. Con el fin de evaluar diferencias significativas en la vegetación e insectos se aplicó análisis de Kruskal wallis y X ${ }^{2}$ para determinar si lo registrado es lo potencialmente presente. Para determinar la posible asociación entre variables del hábitat que determinan la presencia de aves e insectos.

Se aplicaron análisis de regresión Poisson (ARP), mediante procedimiento de selección de variables polinómica Stepwise, el ajuste de los modelos se realizó con el criterio del mínimo Akaike en R.13.0 (Akaike, 1969). Con la finalidad de determinar el grado de asociación entre la abundancia de aves e insectos determinados por las variables del hábitat y vegetación se aplicaron análisis de asociación mediante correspondencia canónica en el software estadístico XLSTAT versión 2018.7.

\section{Resultados y discusión}

Se describieron un total de 495 ejemplares de aves distribuidos en 10 ordenes, 19 familias, 42 géneros y 51 especies, 1741 ejemplares de vegetación agrupados en 38 familias (Figura 1) y 165 insectos, repartidos en 9 ordenes, 40 familias, 51 géneros y 53 especies (Figura 2).

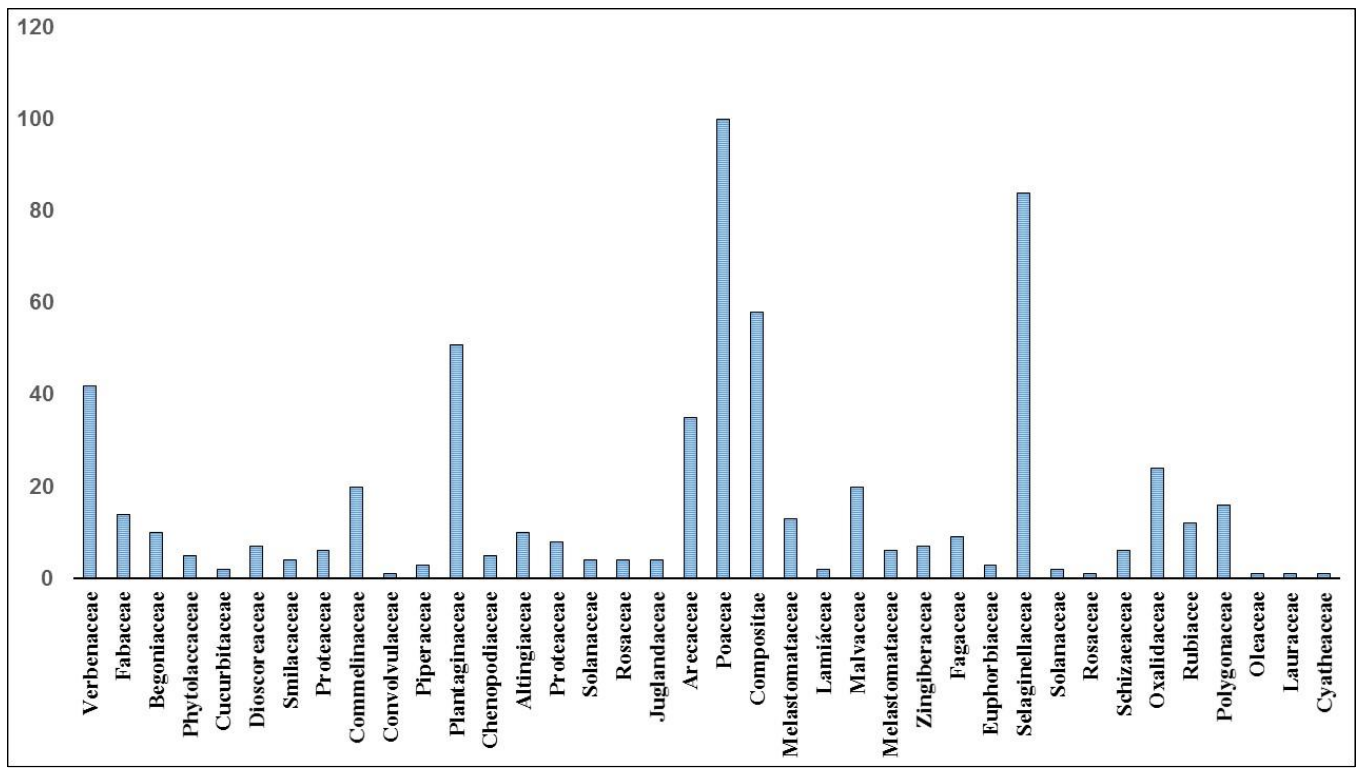

Figura 1. Familias vegetales registradas en las tres condiciones de estudio. 


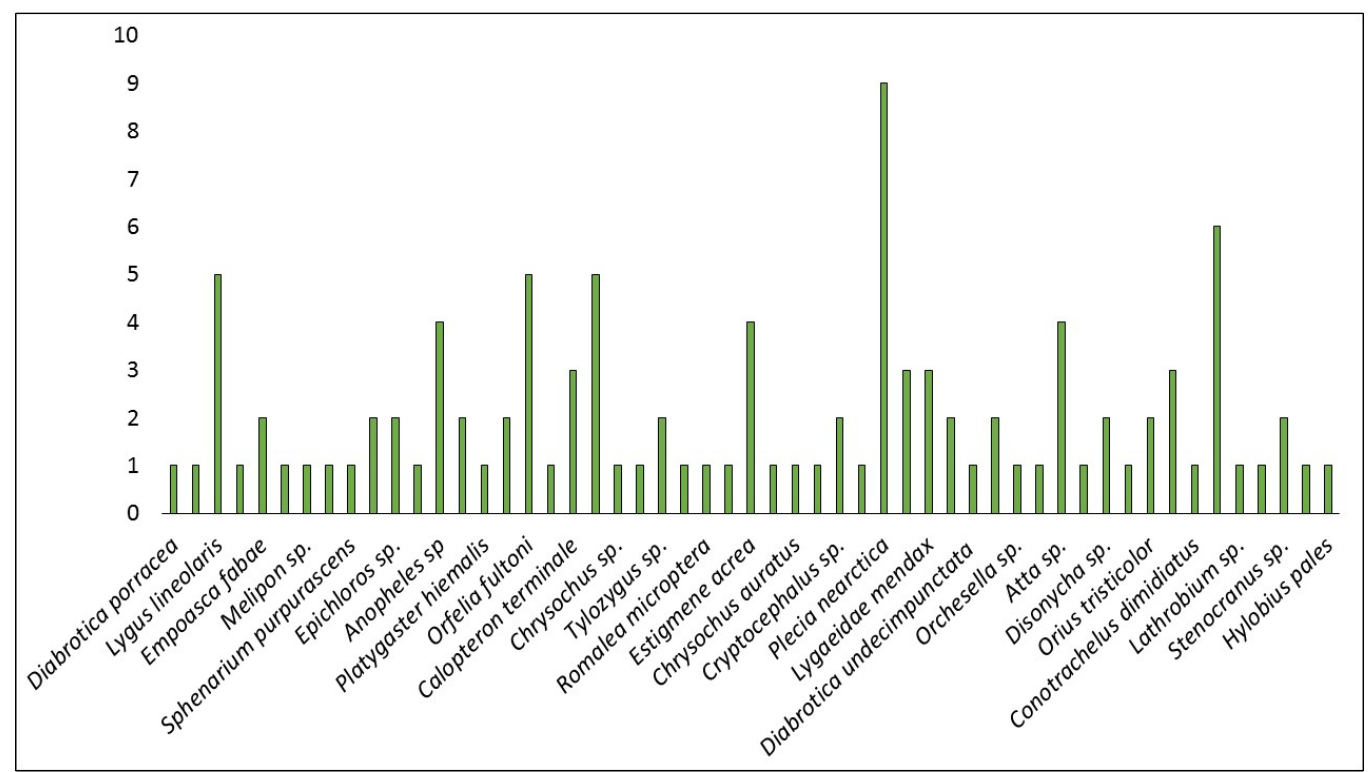

Figura 2. Especies de insectos registrados mediante corte y sacudida en las tres condiciones.

La FO establece valores altos para: Melanerpes aurifrons $(8.31 \%)$; Cyanocorax morio $(7.70 \%$; Figura 3); Poaceae (61.49\%; Figura 4) y Plecia nearctica (8.41\%; Figura 5).

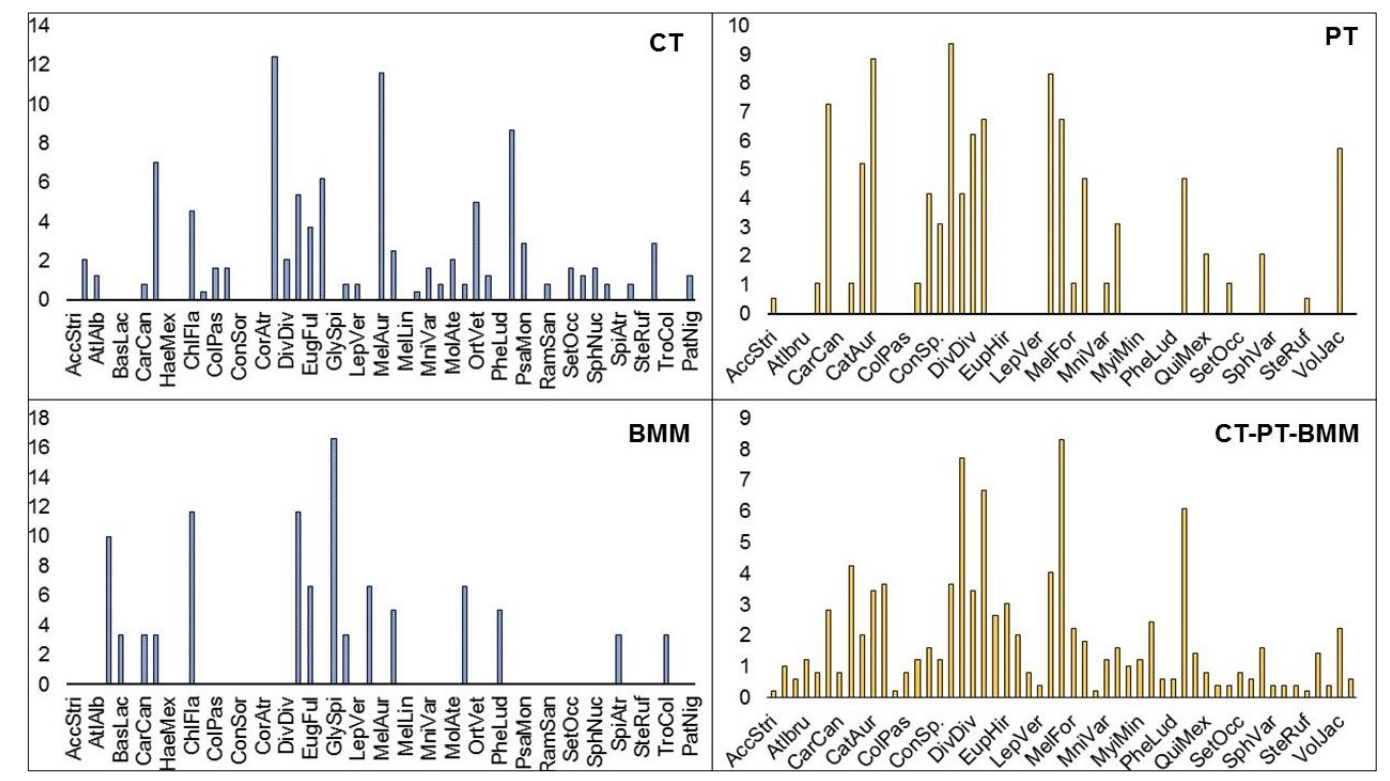

Figura 3. Frecuencia de observación de las aves registradas en las condiciones evaluadas. 


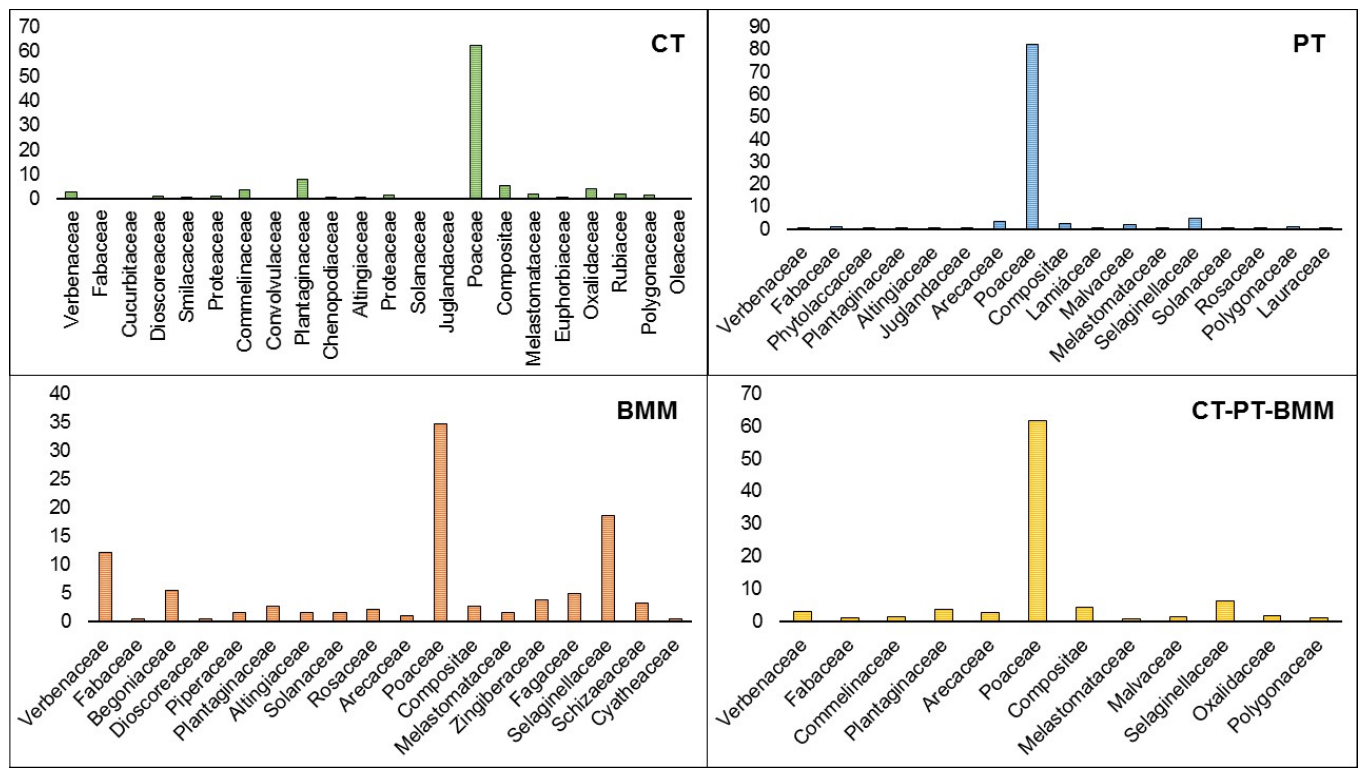

Figura 4. Frecuencia de observación de las familias de vegetación registradas.

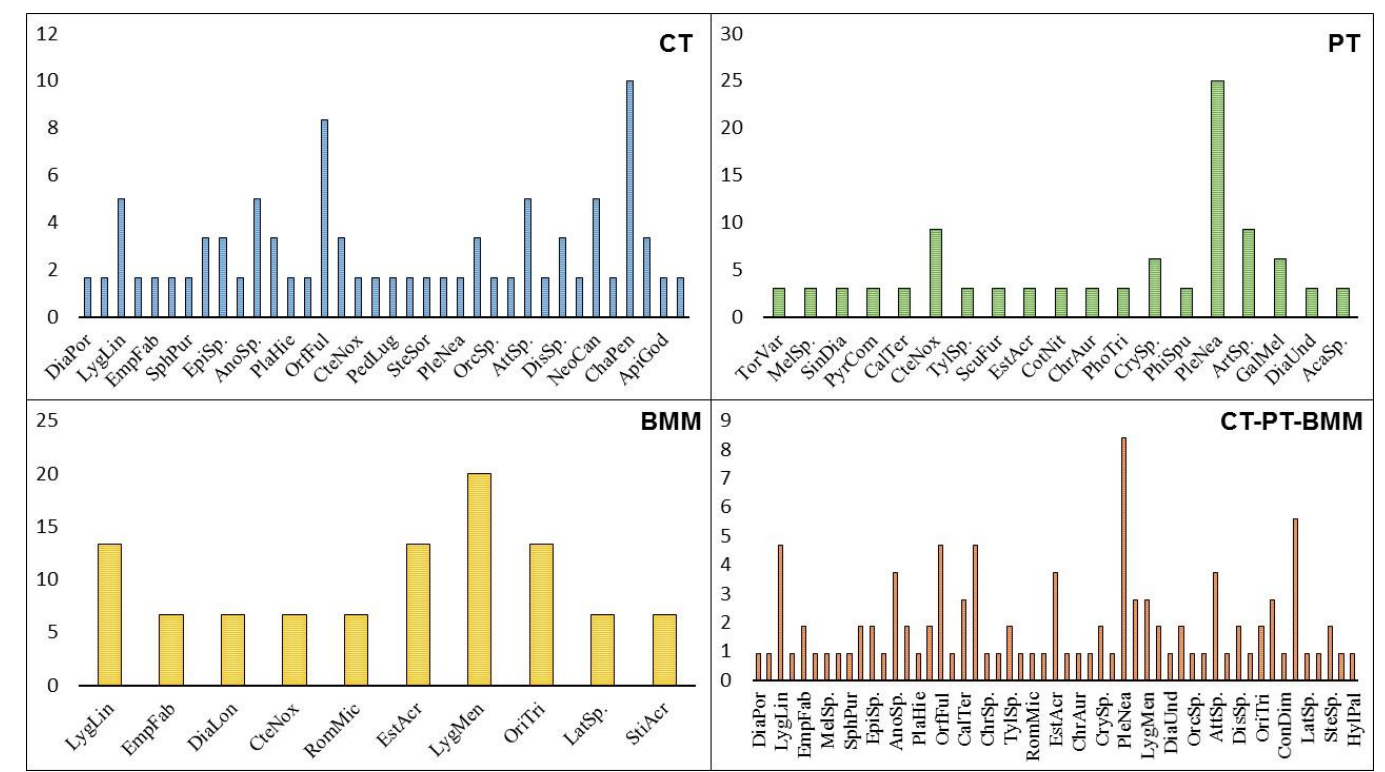

Figura 5. Frecuencia de observación de las especies de insectos registrados.

El IAR señala que la vegetación de mayor abundancia fue Poaceae (0.61). Por su parte los insectos más abundantes fueron: $\mathrm{CT}=$ Chauliognathus pensylvanicus $(0.1) ; \mathrm{PT}=$ Plecia nearctica (0.25); $\mathrm{BMM}=$ Lygaeidae mendax (0.2) y CT-PT-BMM= Plecia nearctica (0.084) (Figura 6). 


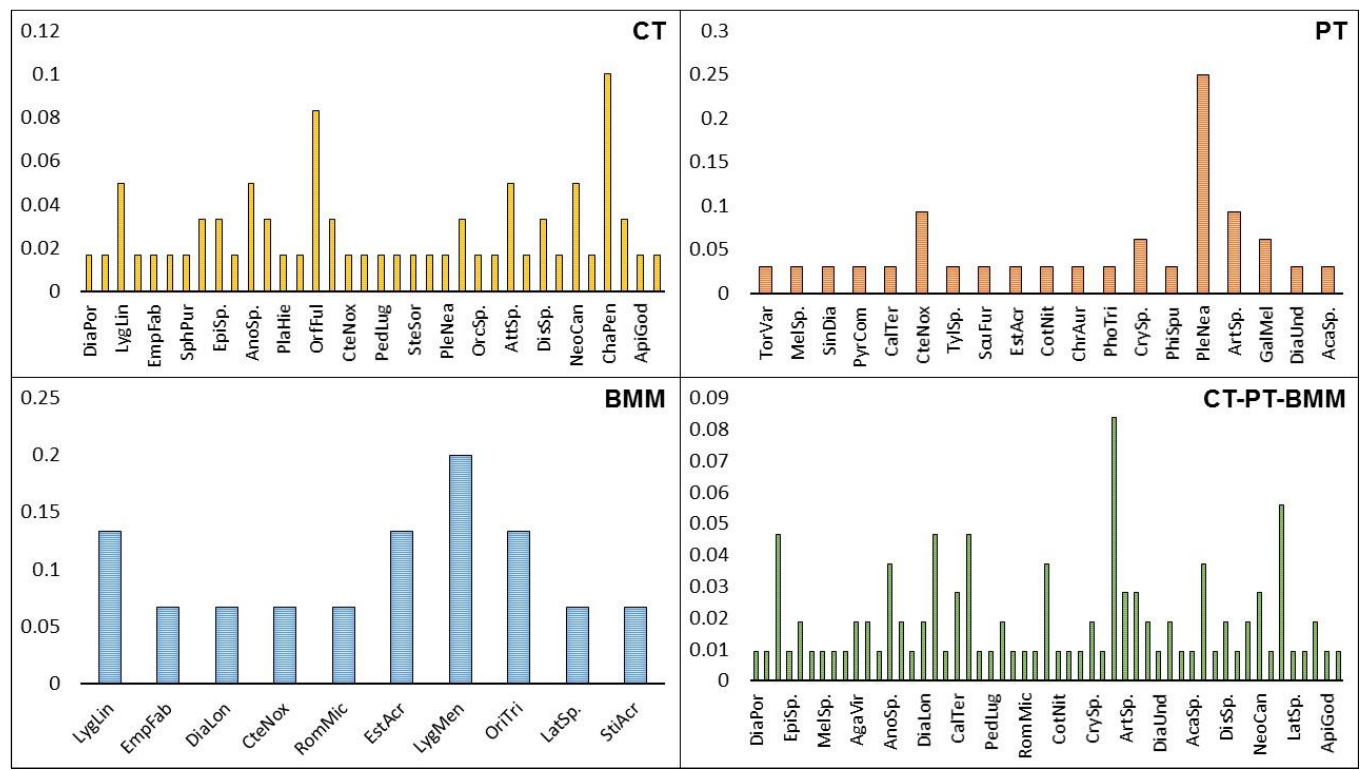

Figura 6. Índice de abundancia relativa de las especies de insectos registrados.

Kruskal wallis evidencia similitud en la vegetación evaluada $(p=0.7961)$ y diferencias en el registro de insectos $\left(p=0.0321^{*}\right)$. $\mathrm{X}^{2}$ establece que la vegetación $(p=0.8452)$ e insectos $(p=0.2392)$ registrados son los que predominan en las condiciones evaluadas. La regresión Poisson evidencia que sólo siete variables presentan un efecto sobre la abundancia de aves y 10 en la presencia de insectos (Cuadro 1).

Cuadro 1. Regresión Poisson para las variables del hábitat y vegetación que determinan la abundancia de aves e insectos.

\begin{tabular}{cccccc}
\hline Coefficients & Estimate & Std. Error & Z Value & $\operatorname{Pr}(>|\mathrm{z}|)$ & Significancia \\
\hline & Variables que determinan la abundancia de aves & & \\
(Intercept) & 1.318439 & 0.04456 & 29.588 & $<2 \mathrm{e}-16$ & $* * *$ \\
Cuerpo de agua & -0.004905 & 0.255097 & -2.53 & 0.01195 & $*$ \\
Cobertura arbórea & -0.004905 & 0.001631 & -3.008 & 0.00287 & $* *$ \\
Cobertura herbácea & -0.006758 & 0.002766 & -2.444 & 0.01515 & $*$ \\
Distancia & 0.039078 & 0.013872 & 2.817 & 0.00519 & $* *$ \\
Melastomataceae & -0.208431 & 0.103539 & -2.013 & 0.04505 & $*$ \\
Poaceae & 0.006529 & 0.003104 & 2.103 & 0.03631 & $*$ \\
Solanaceae & -0.260134 & 0.126041 & -2.064 & 0.03993 & $*$ \\
& Variables que determinan la abundancia de insectos & \\
(Intercept) & 11.3399659 & 2.2316807 & 5.081 & 0.0000222 & $* * *$ \\
Altura de fuste limpio & 0.3241767 & 0.1332116 & 2.434 & 0.021588 & $*$ \\
Altingiaceae & 4.2039426 & 2.00778 & 2.094 & 0.045452 & $*$ \\
Altura & -0.0016319 & 0.0006404 & -2.548 & 0.016596 & $*$ \\
\hline
\end{tabular}




\begin{tabular}{cccccc}
\hline Coefficients & Estimate & Std. Error & Z Value & $\operatorname{Pr}(>|\mathrm{z}|)$ & Significancia \\
\hline Altura $(\mathrm{cm})$ & 0.0206169 & 0.0049136 & 4.196 & 0.000248 & $* * *$ \\
Altura arbórea & -0.5102171 & 0.1612225 & -3.165 & 0.003723 & $* *$ \\
Altura promedio & 0.0419453 & 0.0097174 & 4.316 & 0.000179 & $* * *$ \\
Cobertura arbórea & 0.0484761 & 0.0175949 & 2.755 & 0.010197 & $*$ \\
Cobertura vegetal Arbórea & -0.0855678 & 0.0226578 & -3.777 & 0.000763 & $* * *$ \\
Cobertura vegetal Arbustiva & -0.0801637 & 0.0221586 & -3.618 & 0.001159 & $* *$ \\
Cobertura vegetal herbácea & -0.0847409 & 0.0215242 & -3.937 & 0.000497 & $* * *$ \\
\hline
\end{tabular}

Códigos de significancia $=0$ ‘***’ $0.001^{\text {‘ } * * ’} 0.01^{\text {‘ } * ’ 0} 0.05^{\prime} .0 .1^{\text {‘ } ’} 1$.

El ACC establece una inercia acumulada en sus dos primeros ejes del 84.62\% para aves (Figura 7) y de $87.98 \%$ para insectos (Figura 8). En las figuras, se evidencia como las especies de aves e insectos tienen preferencia por ciertas variables de hábitat y vegetación.

Las tendencias registradas para las variables del hábitat y vegetación que determinan la abundancia de aves e insectos concuerdan con lo señalado por Sánchez et al. (2016) quienes evaluaron la estructura arbórea de un sistema agroforestal (SAF) en Tabasco, México; en dicho estudio señalan que las familias de vegetación más representativas son Fabaceae, Verbenaceae, Moraceae, entre otros, tal como se registró en el presente en el cual se detectaron aves asociadas al estrato arbóreo (con familias de este tipo) alimentándose de insectos, en su mayoría del orden coleóptera y araneae (algunos considerados plaga), se concuerda con Caprio et al. (2015); Figueroa-Sandoval et al. (2019) quienes señalan como la estructura arbórea es un elemento clave en la abundancia de insectos (particularmente carábidos y arañas) que fijan el stock alimenticio de las aves, de acuerdo con Saavedra et al. (2015) este estrato dispone de gran cantidad de coleópteros (particularmente de la familia Scarabaeidae).

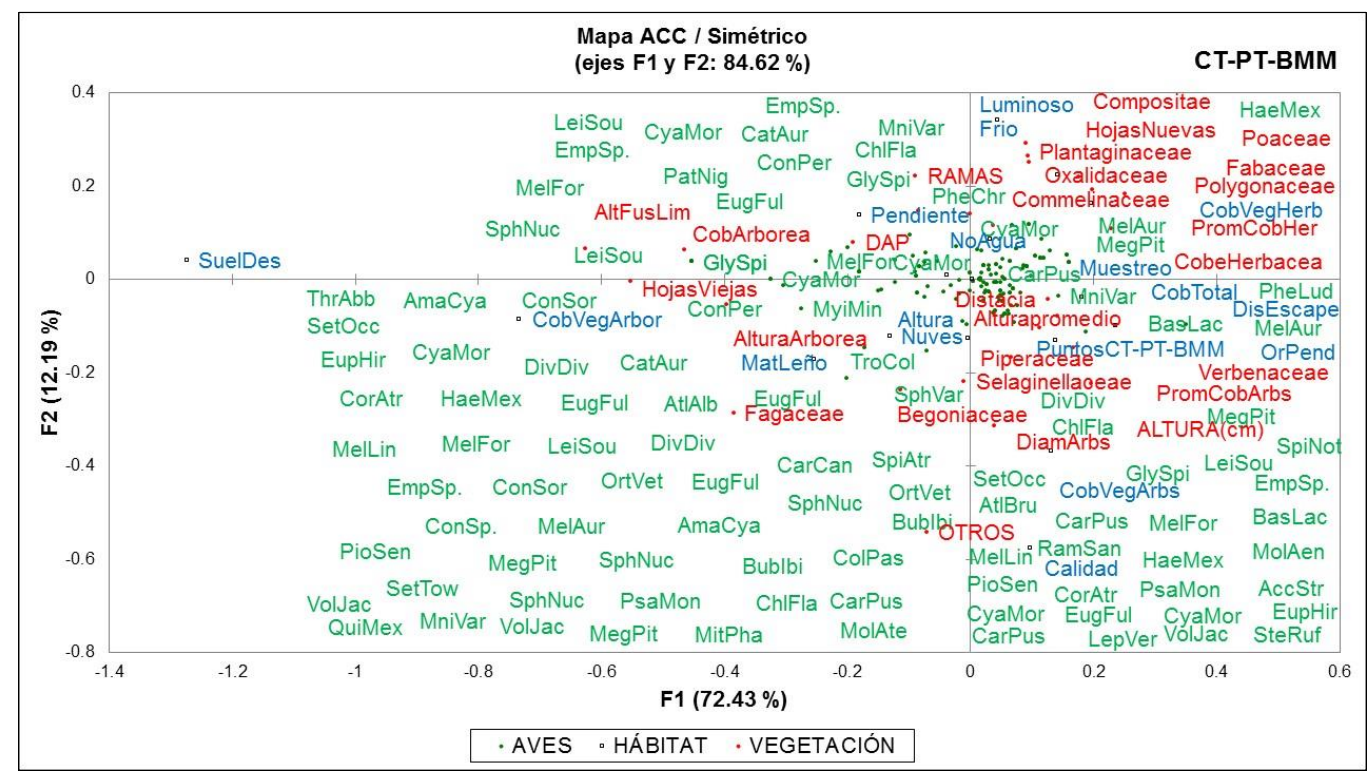

Figura 7. Variables del hábitat y vegetación que determinan la presencia de aves. 


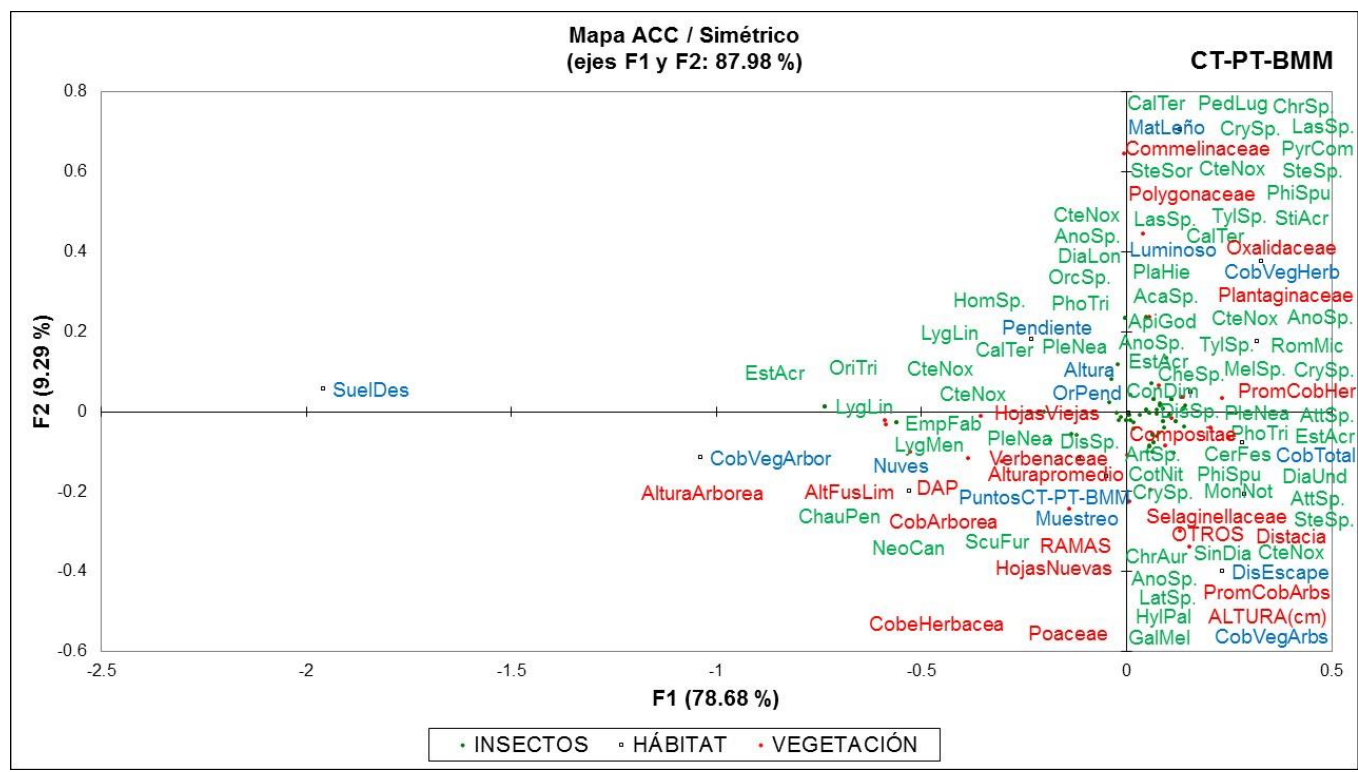

Figura 8. Variables del hábitat y vegetación que determinan la presencia de insectos.

Lo anterior, podría relacionarse con la incidencia solar sobre el dosel, esto debido a que los insectos presentan la característica de ser ectotermos por lo cual requieren de calor para cubrir sus requerimientos mínimos; no obstante patrones de segregación, adaptación y utilización de nichos han ocasionado que ciertos insectos al igual que algunas aves se dispongan en el estrato bajo exhibiendo patrones de coexistencia trófica que permiten una adecuación en el reparto de los recursos.

Se observó cómo especies migratorias de pequeño tamaño se alimentaban sobre la cubierta vegetal del estrato bajo (herbáceas y arbustivas) colectando insectos del orden araneae, coleóptera e himenoptera; de esta forma se puede inferir como la estructura del hábitat determina el acervo alimenticio de la avifauna, tal como lo describen Schaub et al. (2010); Bosco et al. (2019); Bucher et al. (2019) quienes señalan como el estrato bajo dispone de escarabajos y arañas que alimentan a las aves permitiendo un equilibrio en la abundancia de insectos.

En el mismo tenor Morales et al. (2008); Tarjuelo et al. (2017) evidencian patrones de segregación ecológica basada en la estructura vegetal haciendo que ciertas aves utilicen el estrato más bajo para adquirir alimento favoreciendo la coexistencia trófica entre especies; tal como se expone en el análisis de regresión visualizándose individuos de Molothrus aeneus quienes se alimentaban en la cobertura herbácea del PT consumiendo insectos del orden lepidóptera y coleóptera.

Se coincide con Ortega-Rivera et al. (2019) quienes señalan como la apertura de dosel en zonas perturbadas permiten mayor radiación que incrementa la incidencia de insectos que podrían tornarse plaga, pero son regulados por aves y otros grupos taxonómicos (reptiles y anfibios) que mantienen la sanidad vegetal de los ecosistemas.

No obstante, dicho equilibrio no solo se refleja entre las aves sino también por la presencia de insectos paracitos que controlan la incidencia de organismos perjudiciales para la producción agrícola; de esta forma se registraron cuatro insectos parásitos en CT y uno en PT que se 
alimentaban de insectos que podrían ser perjudiciales para ciertas especies forestales, cumpliendo el papel de control biológico; se concuerda con Thomson y Hoffmann (2009) quienes señalan que áreas no cultivadas.

Favorecen a una mayor diversidad de artrópodos que alimentan a diferentes grupos taxonómicos (aves, reptiles, anfibios, insectos parásitos, entre otros) dando un equilibrio en la incidencia de insectos para los diferentes estratos; así Traba et al. (2008); Puig-Montserrat et al. (2017) señalan como la estructura vegetal multiestrato en cultivos orgánicos al igual que los SAFs permite una adecuada distribución de recursos exhibiendo patrones de coexistencia. En donde las aves disponen de diferentes nichos de utilización en sustratos y estratos horizontales y verticales creando condiciones propicias para una mayor diversidad de artrópodos que cumplen el rol de polinizadores y sirven de alimento para la avifauna y otros grupos taxonómicos.

Con forme a esto podemos inferir como los SAFs bien diseñados coadyuvan a la conservación avifaunistica otorgando nichos de alimentación, refugio, sitios de anidación, áreas de descanso, entre otros; así mismo, estas retribuyen a tales beneficios desarrollando funciones ambientales que mantienen el equilibrio ambiental bajo condiciones óptimas exponiendo mejores tazas de producción para los productores. Las diferencias en la estructura vegetal evaluada (CT, PT, BMM) presentan un efecto sobre la abundancia de algunas especies de aves, esto concuerda con Arteaga (2018) quien señala que la estructura y composición vegetal determina la abundancia de aves.

En un sistema agroforestal de pino; así la heterogeneidad vegetal es un factor determinante en la abundancia y distribución de las especies por ello la condición de CT con más estratos hospedo un mayor número de individuos por especie de aves en comparación con PT y BMM, este último se debe a que el presente estudio solo considero la evaluación de aves del estrato bajo y medio teniendo en consideración que el mayor número de especies en bosques maduros se disponen en el estrato superior.

Ciertos autores señalan que la abundancia de especies es mayor en áreas perturbadas en comparación con zonas conservadas, no obstante, el hecho de que un bosque perturbado soporte un mayor número de individuos no implica que sea de mayor importancia para la conservación; de tal forma Van Horne (1983); McArthur et al. (2019); Roberts y King (2019) señalan que la densidad de individuos por sí sola no debe ligarse positivamente con la calidad del hábitat; es necesario, contemplar otros factores como la composición, estructura y dinámica poblacional; señala que entre la diversidad de hábitats y diversidad faunistica, no siempre existe una relación positiva, ya que ésta depende del número de especies generalistas y especialistas, puntualiza la probabilidad de que hábitats más conservados originen un mayor número de individuos y que por efectos territoriales (competencia y segregación por los recursos).

El exceso de estos se dispone en hábitats de menor calidad y sitios con efecto de borde, aunque la abundancia y densidad por si solas no determinan la calidad ambiental, cuando se relacionan con las características de la vegetación permiten generar proyecciones de respuesta sobre las poblaciones de aves convirtiéndose en un instrumento importante para el manejo de hábitat y de las poblaciones que lo utilizan (sean estas especialistas o generalistas). De esta forma Durán y Kattan (2005) señalan que bosques perturbados con vegetación secundaria como CT y PT rodeados de bosque maduro (BMM) no necesariamente presentan un efecto negativo sobre las comunidades de aves y sus procesos ecológicos (competencia, segregación, entre otros), por ello las especies 
generalistas también deben ser objeto de conservación ya que estas aportan a la diversidad general y presentan una mayor capacidad de respuesta a factores de perturbación en comparación con especies raras y especialistas.

Desde el punto de vista ecológico las tres condiciones evaluadas (CT, PT, BMM) resultan importantes en la conservación de este grupo taxonómico. En este contexto las abundancias de aves registradas en cada condición no deben contemplarse como un indicador de la calidad ambiental, su interpretación ecológica debe estar asociada a diferentes factores poblacionales y del hábitat para inferir la calidad de estos sistemas productivos.

Este estudio aporta información relevante sobre los SAFs y bosque conservado (BMM) en relación a la abundancia de aves y su recurso alimenticio (insectos) para esta región particular de Huatusco, Veracruz, México; sin embargo, para diseñar mejores planes de manejo agroforestal y de mejoramiento del hábitat, son necesarios estudios específicos que permitan conocer las implicaciones que presentan estos sistemas sobre los parámetros poblacionales de la avifauna como indicadores de calidad ambiental que permitan la conservación de este y otros grupos taxonómicos.

\section{Conclusiones}

Se logró definir la vegetación y estructura del hábitat en relación con la presencia de aves e insectos presa. Dichos factores son determinantes en la abundancia de aves y disponen del stock alimenticio garantizando la conservación y equilibrio ambiental de los sistemas agroforestales. Este trabajo es uno de los primeros antecedentes para futuros trabajos en donde se aborde la temática sobre vegetación y uso de hábitat que determina el acervo alimenticio y coexistencia avifaunística.

\section{Agradecimientos}

Al Consejo Nacional de Ciencia y Tecnología (CONACYT) por el financiamiento para el desarrollo de este trabajo. A la maestría en Ciencias en Agroforestería para el Desarrollo Sostenible y al Centro Regional Universitario (CRUO) por permitir desarrollar este proyecto en los campos experimentales.

\section{Literatura citada}

Abouhamad, S. de L.; Ramírez, M.; Ramírez, J.; Céspedes, K. y Alpízar, A. 2017. Servicios ecosistémicos de regulación que benefician a la sociedad y su relación con la restauración ecológica. Biocenosis. 31(1-2):80-92.

Akaike, H. 1969. Fitting autoregressive models for prediction. Annals of the Institute of Statistical Mathematics. 21(1):243-217.

Almazán-Núñez, R. C.; Puebla-Olivares, F. y Almazán-Juárez, Á. 2009. Diversidad de aves en bosques de pino-encino del centro de guerrero, México. Acta Zoológica Mexicana. 25(1):123-142.

Alonso, Y.; Hernández, F. R. y Barrero, H. 2017. Diversidad de aves residentes y permanentes asociadas a un pinar natural de Pinus tropicalis y su relación con la estructura vertical de la vegetación. Revista Cubana de Ciencias Forestales. 6(1):31-44.

Arteaga, M. 2018. Estructura y composición de la vegetación de dos pinares de Pinus caribaea Morelet y su relación con la diversidad de las aves asociadas. Revista Científico estudiantil Ciencias Forestales y Ambientales. 3(2):193-206. 
Bosco, L.; Arlettaz, R. and Jacot, A. 2019. Ground greening in vineyards promotes the Woodlark Lullula arborea and their invertebrate prey. Journal of Ornithology. 160(3):799-811.

Bucher, R.; Nickel, H.; Kaib, S.; Will, M.; Carchi, J. and Farwig, N. 2019. Birds and plants as indicators of arthropod species richness in temperate farmland. Ecological Indicators. 103(1):272-279.

Bueno, P.; Sánchez, I.; Velásquez, M. A.; Esquivel, G. y Palomo, M. 2015. Caracterización de la vegetación de una microcuenca ubicada en la parte media de RH36. Agrofaz. 15(2):143-149.

Buitrón-Jurado, G. y Tobar, M. 2007. Posible asociación de la ardilla enana Microsciurus flaviventer (rodentia: sciuridae) y bandadas mixtas de aves en la amazonia ecuatoriana. Mastozoología Neotropical. 14(2):235-240.

Caprio, E.; Nervo, B.; Isaia, M.; Allegro, G. and Rolando, A. 2015. Organic versus conventional systems in viticulture: Comparative effects on spiders and carabids in vineyards and adjacent forests. Agricultural Systems. 136(1):61-69.

Castillo, Y. y Calderón, J. 2017. Plantas usadas por aves en paisajes cafeteros de Nariño, Colombia. Revista de Ciencias Agrícolas. 34(2):3-18.

Cruz, B. B. J. y Lauro, L. M. 2006. Asociación de la riqueza y diversidad de especies de aves y estructura de la vegetación en una selva mediana subperennifolia en el centro de Veracruz, México. Revista Mexicana de Biodiversidad. 77(2):235-249.

Cuento, V. R. 2006. Escalas en ecología: su importancia para el estudio de la selección de hábitat en aves. Hornero. 21(1):1-13.

Durán, S. M. and Kattan, G. H. 2005. A test of the utility of exotic tree plantations for understory birds and food resources in the Colombian Andes. Biotropica. 37(1):129-135.

Figueroa-Sandoval, B.; Pimentel-López, J.; Ugalde-Lezama, S.; Figueroa-Rodríguez, O. L.; Figueroa-Rodríguez, K. A. y Tarango-Arámbula, L. A. 2019. Aves en sistemas agrícolas con labranza de conservación en el centro-norte de México. Revista Mexicana de Ciencias Agrícolas. 22(Especial):31-42.

García, A. 2018. Fauna silvestre alimentaria de la reserva sierra de Montenegro, Morelos, México. Ethnoscientia. 3(1-2):715.

López-Ferrer, U. del C.; Brito-Vega, H.; López-Morales, D.; Salaya-Domínguez, J. M. and GómezMéndez, E. 2017. Papel de Trichoderma en los sistemas agroforestales cacaotal como un agente antagónico. Tropical and Subtropical Agroecosystems. 20(1):91-100.

McArthur, N.; Boulton, R.; Richard, Y. and Armstrong, D. 2019. The role of pine plantations in source-sink dynamics of North Island robins. New Zealand Journal of Ecology. 43(1):33-62.

Mora, J. M.; Batista, A. E. y López, U. L. I. 2019. Regeneración natural en sitios impactados por incendios en la Reserva Biologíca Uyuca, Honduras. CEIBA A Scientific Technical Journal. 842(1):1-10.

Morales, M.; Carriles, T.; Delgado, M. and García de la Morena, E. 2008. Sexual differences in microhabitat selection of breeding little bustards Tetrax tetrax: Ecological segregation based on vegetation structure. Acta oecologica. 34(3):345-353.

Naranjo, L. G. y Ulloa, P. C. 1997. Diversidad de insectos y aves insectivoras de sotobosque en habitats perturbados de selva lluviosa tropical. Caldasia. 19(3):507-520.

Navarro-Sigüenza, A. G.; Rebón-Gallardo, M. F.; Gordillo-Martínez, A.; Peterson, A. T.; Berlanga-García, H. y Sánchez-González, L. A. 2014. Biodiversidad de aves en México. Revista Mexicana de Biodiversidad. 85(12):S476-S495.

Ortega-Rivera, K.; Flores-Hernández, N.; Zarza, H. y Chávez, C. 2019. Caracterización del estado fitosanitario de Quercus obtusata Bonpl., en bosque mesófilo de montaña, Xicotepec, Puebla Phytosanitary characterizaction of Quercus obtusata Bonpl., in a mountain cloud forest, Xicotepec, Puebla. Revista Mexicana de Ciencias Forestales. 10(53):64-85. 
Ponce, L. P.; Aguilar, V. B.; Rodríguez, T. D.; López P. E. y Santillán, P. J. 2012. Influencia del fuego sobre la riqueza y diversidad de aves en un bosque templado en Puebla. Revista Mexicana de Ciencias Forestales. 3(10):65-76.

Puig-Montserrat, X.; Stefanescu, C.; Torre, I.; Palet, J.; Fàbregas, E.; Dantart, J.; Arrizabalaga, A. and Flaquer, C. 2017. Effects of organic and conventional crop management on vineyard biodiversity. Agriculture, Ecosystems and Environment. 243(1):19-26.

Ramírez-Albores, J. E. 2009. Diversidad de aves de hábitats naturales y modificados en un paisaje de la Depresión Central de Chiapas, México. Revista de Biologia Tropical. 58(1):511-528.

Rangel-Salazar, J. L.; Enríquez, P. L. y Sántiz López, E. C. 2009. Variación de la diversidad de Aaes de sotobosque en el parque nacional lagos de Montebello, Chiapas, México. Acta Zoológica Mexicana. 25(3):479-495.

Roberts, P. and King, D. 2019. Variation in plumage reflects avian habitat associations not revealed by abundance. The Wilson Journal of Ornithology. 131(2):339-347.

Saavedra, A. D.; Vaz de Mello, F.; Ugaz, A. y Pacherre, T. C. 2015. Coleópteros (Coleóptera: Scarabaeidae) de los bosques de niebla, Ramos y Chin Chin, Ayabaca-Huancabamba, Piura-Perú. Indes. 3(1):108-116.

Sánchez, G. F.; Pérez-Flores, J.; Obrador, O. J.; Sol, S. Á. y Ruiz-Rosado, O. 2016. Estructura arbórea del sistema agroforestal cacao en Cárdenas, Tabasco, México. Revista Mexicana de Ciencias Agrícolas. 7(14):2695-2709.

Schaub, M.; Martinez, N.; Tagmann-Ioset, A.; Weisshaupt, N.; Maurer, M. L.; Reichlin, T. S. Abadi, F.; Zbinden, N.; Jenni, L. and Arlettaz, R. 2010. Patches of Bare Ground as a Staple Commodity for Declining Ground-Foraging Insectivorous Farmland Birds. PlosOne. 5(10):1-5.

Serial, R. M. B. y Grigera, D. 2005. Dinámica estacional del ensamble de aves de un bosque norpatagónico de lenga (Nothofagus pumilio) y su relación con la disponibilidad de sustratos de alimentación. Hornero. 20(2):131139.

Tarjuelo, R.; Traba, J.; Morales, M. and Morris, D. 2017. Isodars unveil asymmetric effects on habitat use caused by competition between two endangered species. Oikos. 126(1):73-81.

Thomson, L. and Hoffmann, A. 2009. Vegetation increases the abundance of natural enemies in vineyards. Biological Control. 40(3):259-269.

Traba, J.; Morales, M.; García de la Morena, E. and Delgado, M. P. 2008. Selection of breeding territory by little bustard (Tetrax tetrax) males in Central Spain: the role of arthropod availability. Ecological Research. 23(3):615-622.

Tzuc-Martínez, R.; Casanova-Lugo, F.; Caamal-Maldonado, A.; Tun-Garrido, J.; GonzálezVáldivia, N. y Cetzal-Ix, W. 2017. Influencia de las especies leñosas en la dinámica de arvenses en sistemas agroforestales en Yucatán, México. Agrociencia. 51(3):315-328.

Valencia-Trejo, G. M.; Ugalde-Lezama, S.; Pineda-Pérez, F. E.; Tarango-Arámbula, L. A.; Lozano-Osornio, A. y Cruz-Miranda, Y. 2014. Diversidad de aves en el Campus Central de la Universidad Autónoma Chapingo, México. Agropoductividad. 7(5):37-44.

Van Horne, B. 1983. Density as a misleading indicator of habitat quality. Journal of Wildlife Management. 47(4):893-901.

Vazquez-Perez, J. R.; Enríquez-Rocha, P. L. y Rangel-Salazar, J. L. 2009. Diversidad de aves rapaces diurnas en la reserva de la biosfera Selva El Ocote, Chiapas, México. Revista Mexicana de Biodiversidad. 80(1):203-209. 